$$
H^{*}\left(\Omega G_{2}, Z\right) \cong Z[x, h(x), t(x)] \otimes Z\langle y\rangle
$$

where $h(x)=\infty$ and $t(x)$ is defined by the greatest divisors $g\left(x^{n}\right)$ $=n ! / g\left(u^{n}\right)$. In particular, $g\left(x^{2}\right)=1$ so that (1.15) fails for $x^{2}$ and $H^{*}\left(\Omega G_{2}, Z\right)$ has no system of divided powers.

\title{
BIBLIOGRAPHY
}

1. A. Borel, Sur la cohomologie des espaces fibrés principaux et des espaces homogènes de groupes de Lie compacts, Ann. of Math. vol. 57 (1953) pp. 115-207.

2. R. Bott, The loop space on a Lie group, Michigan Math. J. vol. 5 (1958) pp. 35-67.

3. H. Cartan, Séminaire de Topologie de l'Ecole Normale Supérieure, Paris, 19541955, Notes polycopiées.

4. E. Halpern, On the structure of hyperalgebras. Class 1 Hopf algebras, Portugal. Math. vol. 17 (1958) p. 127-147. 1958.

5. —-, Twisted polynomial hyperalgebras, Memoir Amer. Math. Soc., no. 29,

UNIVERSITY OF MrChigaN

\section{ANOTHER CUTPOINT THEOREM FOR PLANE CONTINUA}

\section{F. BURTON JONES ${ }^{1}$}

If the subcontinuum $M$ of a topological 2-sphere $S$ does not separate $S$ and is locally connected, then each pair of points of $M$, which are not separated in $M$ by a point of $M$, belongs to the closure of a connected domain (of $S$ ) lying in $M$. This is true because each such pair of points belongs to a simple closed curve $J$ lying in $M$ and one of the complementary domains of $J$ is a subset of $M$. However, without local connectedness such a simple closed curve may fail to exist. In fact, the proposition would then be false because (to take an extreme case) of the existence of indecomposable subcontinua of $S$ which fail to separate $S$. While no point of an indecomposable continuum separates it, every point of it cuts it. Recently I showed [1] that this stronger form of separation (or rather the lack of it) is sufficient to restore the validity of the above proposition in the absence of local connectedness if a certain restriction were placed upon

Presented to the Society, August 29, 1958; received by the editors September 15, 1959.

${ }^{1}$ This research was supported (in part) by the United States Air Force through the Air Force Office of Scientific Research of the Air Research and Development Command, under contract No. AF18(600)-1109. Reproduction in whole or in part is permitted for any purpose of the United States Government. 
the pair of points under consideration. It is the purpose of this paper to remove even this restriction.

NOTATION AND TERMINOLOGY. If $p$ and $q$ are points of a continuum $M$ and $x$ is a point of $M-(p+q), x$ is said to $c u t p$ from $q$ in $M$ if every subcontinuum of $M$ which contains $p+q$ contains $x$. By an interior point of $M$ is meant a nonboundary point of $M$. By "the plane" is meant the Euclidean number plane with $d$ denoting the usual Pythagorean distance function.

Theorem. Suppose that $M$ is a compact subcontinuum of the plane $S$ which does not separate $S$. If no point of $M$ cuts the point $p$ from the point $q$ in $M$ then some component of the set of interior points of $M$ contains both $p$ and $q$ in its closure.

INDICATION OF PROOF. If either $p$ or $q$ is an interior point of $M$, the theorem follows from a previous result [1]. So we have left to prove the theorem for the case when both $p$ and $q$ are boundary points of $M$.

Suppose that $\epsilon$ is a positive number such that $2 \epsilon<d(p, q)$. Let $C_{p}(\epsilon)$ and $C_{q}(\epsilon)$ denote circles of radius $\epsilon$ centered on $p$ and $q$ respectively. There exists a simple domain $I(\epsilon)$ which contains $M$ such that if $J(\epsilon)$ denotes the boundary of $I(\epsilon), y$ is a boundary point of $M$ and $z$ is a point of $I(\epsilon)+J(\epsilon)$ then $d[y, J(\epsilon)]<\epsilon$ and $d(z, M)<\epsilon$. There exist $\operatorname{arcs} T_{p}(\epsilon)$ and $T_{q}(\epsilon)$ in $C_{p}(\epsilon)$ and $C_{q}(\epsilon)$ respectively such that each is minimal with respect to separating $p$ from $q$ in $I(\epsilon)+J(\epsilon)$ and $T_{p}(\epsilon)$ separates $p$ from $q+T_{q}(\epsilon)$ in $I(\epsilon)+J(\epsilon)$. It follows that $T_{q}(\epsilon)$ separates $p+T_{p}(\epsilon)$ from $q$ in $I(\epsilon)+J(\epsilon)$.

Since $T_{p}(\epsilon)$ and $T_{q}(\epsilon)$ have only their endpoints in $J(\epsilon)$, there exist in $J(\epsilon)$ two nonintersecting arcs $A(\epsilon)$ and $B(\epsilon)$ such that $T_{p}(\epsilon)+A(\epsilon)$ $+T_{q}(\boldsymbol{\epsilon})+B(\epsilon)$ is a simple closed curve $H(\epsilon)$. Let $D(\epsilon)$ denote the bounded complementary domain of $H(\epsilon)$. If $z$ is a point of $D(\epsilon)+H(\epsilon)$, then $d(z, M)<\epsilon$. Any subcontinuum of $M$ which contains $p+q$ contains a subcontinuum irreducible from $T_{p}(\epsilon)$ to $T_{q}(\epsilon)$ which lies in $T_{p}(\boldsymbol{\epsilon})+D(\epsilon)+T_{q}(\boldsymbol{\epsilon})$.

Now let $L(\epsilon)$ denote a continuum lying in $T_{p}(\epsilon)+D(\epsilon)+T_{q}(\epsilon)$ which intersects both $T_{p}(\epsilon)$ and $T_{q}(\epsilon)$ such that if $z$ belongs to $L(\epsilon)$, then $d[z, A(\epsilon)]=d[z, B(\epsilon)]$. There exists a simple infinite sequence $\alpha$ of values of $\epsilon$ such that $D(\epsilon)+H(\epsilon)$ converges to a subset of $M$ and $L(\epsilon) \rightarrow L$ as $\epsilon \rightarrow 0$ in $\alpha$. The set $L$ has the following properties:

(a) $L$ is a continuum containing both $p$ and $q$,

(b) $L$ is a subset of $M$, and

(c) every point of $L-(p+q)$ is an interior point of $M$. Properties (a) and (b) are evident. So it remains only to prove property (c).

Let $x$ be a point of $L-(p+q)$. Since $x$ does not cut $p$ from $q$ in 
$M$, there exists a subcontinuum $K$ of $M$ which contains $p+q$ but not $x$. Let $\delta$ be a positive number such that $4 \delta=d(x, K)$ and let $U_{\delta}(x)$ and $U_{3 \delta}(x)$ be the circular regions centered on $x$ of radius $\delta$ and $3 \delta$ respectively. When $\epsilon$ (in $\alpha$ ) is sufficiently small $\left[T_{p}(\epsilon)+T_{q}(\epsilon)\right]$ - $\left[U_{3 \delta}(x)\right]=0$ but $L(\epsilon) \cdot U_{\delta}(x) \neq 0$. Let $y$ be some point of $L(\epsilon) \cdot U_{\delta}(x)$, let $r=\delta+d(x, y)$ and let $U_{r}(y)$ be a circular region of radius $r$ and center $y$. Obviously $U_{3 \delta}(x) \supset U_{r}(y) \supset U_{\delta}(x)$. So $\left[T_{p}(\epsilon)+T_{q}(\epsilon)\right] \cdot U_{r}(y)$ $=0$. If $A(\epsilon) \cdot U_{r}(y) \neq 0$, let $f$ be a point of $A(\epsilon) \cdot U_{r}(y)$ such that $d(f, y)=d[y, A(\epsilon)]$. But $y$ belongs to $L(\epsilon)$. Hence there exists in $U_{r}(y)$ a point $g$ of $B(\epsilon)$ such that $d(g, y)=d[g, B(\epsilon)]=d(f, y)$. The sum of the straight line intervals from $y$ to $f$ and from $y$ to $g$ is an arc $T_{y}$ lying in $U_{r}(y)$, having only its endpoints $f$ and $g$ in $H(\epsilon)$, and containing the point $y$ of $D(\epsilon)$. Hence $T_{y}-(f+g) \subset D(\epsilon)$. But $T_{y} \cdot K=0$ and $K$ contains a continuum lying in $T_{p}(\epsilon)+D(\epsilon)+T_{q}(\epsilon)$ irreducible from $T_{p}(\boldsymbol{\epsilon})$ to $T_{q}(\boldsymbol{\epsilon})$. Since the points $f$ and $g$ separate $T_{p}(\boldsymbol{\epsilon})$ from $T_{q}(\epsilon)$ in $H(\epsilon)$ this involves a contradiction [2, Theorem 17, p. 167]. Hence $U_{r}(y) \cdot H(\epsilon)=0$ and since $y$ belongs to $D(\epsilon), U_{r}(y) \subset D(\epsilon)$; so for sufficiently small values of $\epsilon$ (in $\alpha$ ), $U_{\delta}(x) \subset D(\epsilon)$. Consequently $U_{\delta}(x)$ is a subset of $M$ and $x$ is an interior point of $M$.

The continuum $L$ contains a subcontinuum $N$ which is irreducible from $p$ to $q ; N-(p+q)$ is connected and each of its points is an interior point of $M$. The component of the set of interior points of $M$ which contains $N-(p+q)$ has both $p$ and $q$ in its closure.

Counterexample. The converse of the theorem is false. Let $L$ be the closure of the graph of $y=\sin 1 / x(-\pi \leqq x \leqq \pi)$ together with one arc (the lower one) of the square whose vertices are $( \pm \pi, \pm \pi)$ so that this arc joins the endpoints of the graph, and let $M$ denote $L$ together with its bounded complementary domain $D$. Obviously $\bar{D}=M$ but $(0,-1)$ cuts $(0,-\pi)$ from $(0,1)$ in $M$.

\section{BIBLIOGRAPHY}

1. F. B. Jones, On the existence of weak cut points in plane continua, Proc. Amer. Math. Soc. vol. 9 (1958) pp. 530-532.

2. R. L. Moore, Foundations of point set theory, Amer. Math. Soc. Colloquium Publications, vol. 13, New York, 1932.

The Institute for Advanced Study and The University of North Carolina 